\title{
EXTENDING THE STIELTJES TRANSFORM
}

\author{
DENNIS NEMZER
}

\begin{abstract}
The classical Stieltjes transform is extended to a subspace of Boehmians. The transform is shown to be an analytic function in the half-plane $\operatorname{Re} z>0$. Some Abelian type theorems are established.
\end{abstract}

\section{INTRODUCTION}

The Stieltjes transform was first introduced by T.S. Stieltjes [15] in connection to the moment problem for a semi-infinite interval. Since then it has been investigated and found to be useful in many different areas such as continued fractions, probability, and signal processing, to name a few.

Several authors (see [1]) have extended the classical Stieltjes transform onto spaces of generalized functions.

The space of generalized functions known as Boehmians, whose construction is algebraic, has been used to extend integral transforms such as Fourier, Laplace, Hilbert, and Hankel. Roopkumar [13] has extended the Stieltjes transform onto a space of Boehmians. However, the transform is a Boehmian, not a function.

In this note, we extend the Stieltjes transform onto a subspace of Boehmians by iteration of the Laplace transform. In this case, the transform is an analytic function in the half-plane $\operatorname{Re} z>0$. This allows, in a natural way, to establish some Abelian type theorems.

This article is organized as follows. Section 2 contains notation and the construction of the space of Boehmians. Section 3 is concerned with the Stieltjes transform for Boehmians. First, a brief review of the Laplace transform for Boehmians is given. Then, the Stieltjes transform for Boehmians is defined and some of its properties are established. The inversion for the Stieltjes transform is discussed in Section 4. In Section 5, an initial value, as well as a final value, theorem for the Stieltjes transform is established. In the last section, Section 6, some open problems are presented.

2010 Mathematics Subject Classification. 44A15, 46F12, 44A10.

Key words and phrases. Abelian theorems, Boehmians, generalized function, iterated Laplace transform, Stieltjes transform. 


\section{Preliminaries}

Let $C(\mathbb{R})$ denote the space of all continuous complex-valued functions on the real line $\mathbb{R}$, and let $C^{\infty}(\mathbb{R})$ be the subspace of $C(\mathbb{R})$ of all infinitely differentiable functions.

A sequence of smooth nonnegative functions $\left\{\varphi_{n}\right\}$ is called a delta sequence provided:

(i) $\int_{-\infty}^{\infty} \varphi_{n}(t) d t=1$ for $n=1,2, \ldots$,

(ii) $\operatorname{supp} \varphi_{n} \subset\left(-\varepsilon_{n}, \varepsilon_{n}\right), \varepsilon_{n} \rightarrow 0$ as $n \rightarrow \infty\left(\varepsilon_{n}>0\right)$.

A pair of sequences $\left(f_{n}, \varphi_{n}\right)$ is called a quotient of sequences if $f_{n} \in$ $C^{\infty}(\mathbb{R})$ for $n \in \mathbb{N},\left\{\varphi_{n}\right\}$ is a delta sequence, and $f_{m} * \varphi_{k}=f_{k} * \varphi_{m}$ for all $k, m \in \mathbb{N}$, where $*$ denotes convolution:

$$
(f * \varphi)(t)=\int_{-\infty}^{\infty} f(t-\sigma) \varphi(\sigma) d \sigma .
$$

Two quotients of sequences $\left(f_{n}, \varphi_{n}\right)$ and $\left(g_{n}, \psi_{n}\right)$ are said to be equivalent if $f_{k} * \psi_{m}=g_{m} * \varphi_{k}$ for all $k, m \in \mathbb{N}$. A straightforward calculation shows that this is an equivalence relation. The equivalence classes are called Boehmians. The space of all Boehmians will be denoted by $\beta(\mathbb{R})$ and a typical element of $\beta(\mathbb{R})$ will be written as $W=\left[\frac{f_{n}}{\varphi_{n}}\right]$.

The operations of addition, scalar multiplication, shifting, and differentiation are defined as follows:

$$
\begin{gathered}
{\left[\frac{f_{n}}{\varphi_{n}}\right]+\left[\frac{g_{n}}{\psi_{n}}\right]=\left[\frac{f_{n} * \psi_{n}+g_{n} * \varphi_{n}}{\varphi_{n} * \psi_{n}}\right],} \\
\alpha\left[\frac{f_{n}}{\varphi_{n}}\right]=\left[\frac{\alpha f_{n}}{\varphi_{n}}\right], \text { where } \alpha \in \mathbb{C}, \\
\tau_{a}\left[\frac{f_{n}}{\varphi_{n}}\right]=\left[\frac{\tau_{a} f_{n}}{\varphi_{n}}\right], \text { where }\left(\tau_{a} f\right)(t)=f(t-a), \\
D\left[\frac{f_{n}}{\varphi_{n}}\right]=\left[\frac{f_{n} * \varphi_{n}^{\prime}}{\varphi_{n} * \varphi_{n}}\right] .
\end{gathered}
$$

Define the map $\iota: C(\mathbb{R}) \rightarrow \beta(\mathbb{R})$ by

$$
\iota(f)=\left[\frac{f * \varphi_{n}}{\varphi_{n}}\right]
$$

where $\left\{\varphi_{n}\right\}$ is any fixed delta sequence.

It is not difficult to show that the mapping $\iota$ is an injection which preserves the algebraic properties of $C(\mathbb{R})$. Thus, $C(\mathbb{R})$ can be identified with a proper subspace of $\beta(\mathbb{R})$. Likewise, the space of Schwartz distributions $\mathcal{D}^{\prime}(\mathbb{R})[16]$ can be identified with a proper subspace of $\beta(\mathbb{R})$. Using this identification, 
the Dirac measure $\delta$ corresponds to the Boehmian $\left[\frac{\varphi_{n}}{\varphi_{n}}\right]$, where $\left\{\varphi_{n}\right\}$ is any delta sequence.

Definition 2.1. Let $W_{n}, W \in \beta(\mathbb{R})$ for $n=1,2, \ldots$. We say that the sequence $\left\{W_{n}\right\}$ is $\delta$-convergent to $W$ if there exists a delta sequence $\left\{\varphi_{n}\right\}$ such that for each $k$ and $n, W_{n} * \varphi_{k} \in C(\mathbb{R})$, and for each $k, W_{n} * \varphi_{k} \rightarrow$ $W * \varphi_{k}$ uniformly on compact sets as $n \rightarrow \infty$. This will be denoted by $\delta-\lim _{n \rightarrow \infty} W_{n}=W$.

Definition 2.2. A Boehmian $W$ is said to vanish on an open set $\Omega \subset \mathbb{R}$, denoted $W(t)=0$ on $\Omega$, provided that there exists a delta sequence $\left\{\varphi_{n}\right\}$ such that $W * \varphi_{n} \in C(\mathbb{R}), n \in \mathbb{N}$, and $W * \varphi_{n} \rightarrow 0$ uniformly on compact subsets of $\Omega$ as $n \rightarrow \infty$.

The support of the Boehmian $W$ is the complement of the largest open set on which $W$ vanishes. $W \in \beta^{+}(\mathbb{R})$ provided that supp $W \subseteq[0, \infty)$. $\beta_{c}(\mathbb{R})$ denotes the space of all Boehmians with compact support. If $W=$ $\left[\frac{f_{n}}{\varphi_{n}}\right] \in \beta_{c}(\mathbb{R})$, and $\mathcal{O} \subset \mathbb{R}$ is an open set such that supp $W \subset \mathcal{O}$, then supp $f_{n} \subset \mathcal{O}$ for all but a finite number of $n$ 's.

The subspace of $\beta_{c}(\mathbb{R})$ whose elements are supported on $[0, \infty)$ is denoted by $\beta_{c}^{+}(\mathbb{R})$. That is,

$$
\beta_{c}^{+}(\mathbb{R})=\beta_{c}(\mathbb{R}) \cap \beta^{+}(\mathbb{R}) .
$$

\section{STIELTJES TRANSFORM}

The Stieltjes transform of index $r$ of a suitably restricted function $f$ is given by

$$
S_{z}^{r} f=\int_{0}^{\infty} \frac{f(t) d t}{(z+t)^{r+1}}, z \in \mathbb{C} \backslash(-\infty, 0] .
$$

Let $T \in J^{\prime}(r), r>-1$. That is, $T \in \mathcal{D}^{\prime}(\mathbb{R})$ such that $T=D^{k} f$, where $k \in \mathbb{N}, D$ is the differentiation operator in $\mathcal{D}^{\prime}(\mathbb{R}), f \in L_{\text {loc }}^{1}(\mathbb{R}), \operatorname{supp} f \subseteq$ $[a, \infty)$ for some $a \geq 0$, and $f(t) t^{-r-k+\alpha}$ is bounded as $t \rightarrow \infty$ (for some $\alpha>0)$. Then, the Stieltjes transform of $T$, which is an analytic function in the region $\mathbb{C} \backslash(-\infty, 0]$, is given by $S_{z}^{r} T=\left\langle T_{t},(z+t)^{-r-1}\right\rangle$, for $z \in \mathbb{C} \backslash(-\infty, 0]$. Several authors $([3,7,9,11,12])$ have used the space $J^{\prime}(r)$ to investigate the Stieltjes transform for distributions.

In this section we consider the space of Boehmians $B_{r}(\mathbb{R})$ which is a subspace of $\beta^{+}(\mathbb{R})$ and contains $J^{\prime}(r)$ as a proper subspace. By using what has been developed previously for the Laplace transform for Boehmians [8], the Stieltjes transform $\Lambda_{(\cdot)}^{r}$ is defined on the space $B_{r}(\mathbb{R})$ by iteration of the Laplace transform. For $r>-1$ and $W \in B_{r}(\mathbb{R}), \Lambda_{z}^{r} W$ is an analytic function in the half-plane Re $z>0$. Before defining the Stieltjes transform 
for a Boehmian, we give a brief introduction for the Laplace transform of a Boehmian.

Definition 3.1. Let $W \in \beta^{+}(\mathbb{R}), f \in L_{l o c}^{1}(\mathbb{R})$ (the space of locally integrable functions), and $k \in \mathbb{N}$. Then, $W(t)=D^{k} f(t)$ on $(a, b)$, provided there exists a delta sequence $\left\{\varphi_{n}\right\}$ such that $W * \varphi_{n} \in C(\mathbb{R})(n \in \mathbb{N})$ and $W * \varphi_{n}-D^{k} f *$ $\varphi_{n} \rightarrow 0$ uniformly on compact subsets of $(a, b)$ as $n \rightarrow \infty$.

A Boehmian $W$ is in $B_{r}(\mathbb{R})$ provided $W \in \beta^{+}(\mathbb{R})$ and $W(t)=D^{k} f(t)$ as $t \rightarrow \infty$ for some $k \in \mathbb{N}$, where $f \in L_{\text {loc }}^{1}(\mathbb{R})$ such that supp $f \subseteq[a, \infty)$ (some $a \geq 0$ ) and $f(t) t^{-r-k+\alpha}$ is bounded as $t \rightarrow \infty$ for some $\alpha>0$. That is, $W=V+D^{k} f$, where supp $V \subseteq[0, a]$, and supp $f \subseteq[a, \infty)$ such that $f(t) t^{-r-k+\alpha}$ is bounded as $t \rightarrow \infty$.

The Laplace transform of a Boehmian $W=\left[\frac{f_{n}}{\varphi_{n}}\right] \in B_{r}(\mathbb{R})$ is given by

$$
\widehat{W}(z)=\lim _{n \rightarrow \infty} \widehat{f}_{n}(z)=\lim _{n \rightarrow \infty} \int_{-\infty}^{\infty} e^{-z t} f_{n}(t) d t, \operatorname{Re} z>0 .
$$

\section{Remarks 3.2.}

1. The Laplace transform operator on $B_{r}(\mathbb{R})$ has many of the same properties as the classical Laplace transform (see [8]).

2. The Laplace transform for a Boehmian $W$ is independent of the representative.

3. $\widehat{W}$ is an analytic function in the half-plane $\operatorname{Re} z>0$. Moreover, for each $\varepsilon>0, \widehat{W}(t)=O\left(e^{\varepsilon t}\right)$ as $t \rightarrow \infty$. If $W \in \beta_{c}^{+}(\mathbb{R})$ such that $\operatorname{supp} W \subseteq[0, \sigma]$, then $\widehat{W}$ is an entire function of exponential type at most $\sigma$.

4. Let $W \in J^{\prime}(r)$. Since $J^{\prime}(r) \subset B_{r}(\mathbb{R})$, the Laplace transform of $W$ exists as an element of $J^{\prime}(r)$ and as an element of $B_{r}(\mathbb{R})$. The Laplace transform is consistent on $J^{\prime}(r)$. That is, these two notions agree on $J^{\prime}(r)$.

The Stieltjes transform for $W=\left[\frac{f_{n}}{\varphi_{n}}\right] \in B_{r}(\mathbb{R})$ and $r>-1$ is given by

$$
\Lambda_{z}^{r} W=\frac{1}{\Gamma(r+1)} \int_{0}^{\infty} e^{-z t} t^{r} \widehat{W}(t) d t, \operatorname{Re} z>0,
$$

where $\Gamma$ is the gamma function.

Remark 3.3. For $r>-1, \Lambda_{(\cdot)}^{r}$ is a linear injective mapping from $B_{r}(\mathbb{R})$ into the space of analytic functions in the half-plane $\operatorname{Re} z>0$. Throughout the sequel, unless otherwise stated, $r>-1$.

Now, $J^{\prime}(r) \subset B_{r}(\mathbb{R})$. Therefore, each element of $J^{\prime}(r)$ has a Stieltjes transform as an element of $J^{\prime}(r)$ and also as an element of $B_{r}(\mathbb{R})$. The two 
agree in the half-plane $\operatorname{Re} z>0$. That is, the following diagram commutes, where $\mathcal{A}$ denotes the space of analytic functions in the half-plane $\operatorname{Re} z>0$.

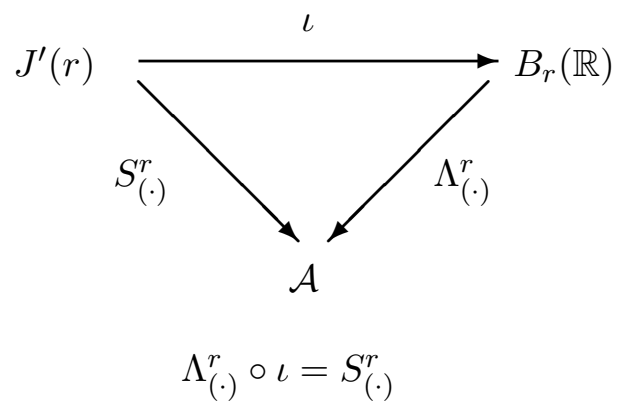

Theorem 3.4. Let $W \in B_{r}(\mathbb{R})$. Then, for $r>-1, \Lambda_{z}^{r} W$ is analytic in the half-plane Re $z>0$.

Proof. Since $W \in B_{r}(\mathbb{R})$, there exist $k \in \mathbb{N}, V \in \beta_{c}^{+}(\mathbb{R})$, and $f \in L_{\text {loc }}^{1}(\mathbb{R})$ with $f(t) t^{-r-k+\alpha}$ bounded as $t \rightarrow \infty$ (some $\alpha>0$ ) such that

$$
W=V+D^{k} f .
$$

Therefore, there exist positive constants $A$ and $B$ such that

$$
|\widehat{f}(t)| \leq A+B t^{-r-k-1+\alpha}, t>0
$$

Thus,

and, for each $\varepsilon>0$,

$$
t^{r}\left(D^{k} f\right)^{\wedge}(t)=t^{r+k} \widehat{f}(t) \in L_{l o c}^{1}\left(\mathbb{R}_{+}\right)
$$

$$
t^{r}\left(D^{k} f\right)^{\wedge}(t)=O\left(e^{\varepsilon t}\right) \text { as } t \rightarrow \infty .
$$

Now, since $V \in \beta_{c}^{+}(\mathbb{R}), \widehat{V}$ is entire, and for each $\varepsilon>0$,

$$
\widehat{V}(t)=O\left(e^{\varepsilon t}\right) \text { as } t \rightarrow \infty \text {. }
$$

Thus,

$$
t^{r} \widehat{V}(t) \in L_{l o c}^{1}\left(\mathbb{R}_{+}\right)
$$

and, for each $\varepsilon>0$,

$$
t^{r} \widehat{V}(t)=O\left(e^{\varepsilon t}\right) \text { as } t \rightarrow \infty .
$$

Therefore,

$$
\begin{aligned}
\Lambda_{z}^{r} W & =\frac{1}{\Gamma(r+1)} \int_{0}^{\infty} e^{-z t} t^{r} \widehat{W}(t) d t \\
& =\frac{1}{\Gamma(r+1)} \int_{0}^{\infty} e^{-z t} t^{r}\left(\widehat{V}(t)+\left(D^{k} f\right)^{\wedge}(t)\right) d t
\end{aligned}
$$


is analytic in the half-plane $\operatorname{Re} z>0$.

We now show that for $W \in B_{r}(\mathbb{R}), \Lambda_{z}^{r} W$ can be analytically extended to a region containing the half-plane $\operatorname{Re} z>0$.

For $\sigma \geq 0, \tilde{B}_{\sigma}(0)=\{z \in \mathbb{C}:|z| \leq \sigma, \operatorname{Re} z \leq 0\}$ and $\mathcal{A}_{\sigma}=\tilde{B}_{\sigma}(0) \cup$ $(-\infty, 0)$.

Theorem 3.5. Let $V \in \beta_{c}(\mathbb{R})$ such that supp $V \subseteq[0, \sigma]$. Then, $\Lambda_{z}^{r} V$ can be analytically extended to the region $\mathbb{C} \backslash \mathcal{A}_{\sigma}$.

Proof. Let $V \in \beta_{c}(\mathbb{R})$ such that $\operatorname{supp} V \subseteq[0, \sigma]$. Then, $\widehat{V}$ is an entire function of exponential type at most $\sigma$. Let

$$
\widehat{V}(z)=\sum_{n=0}^{\infty} c_{n} z^{n}, z \in \mathbb{C} .
$$

Then,

$$
\varlimsup_{n \rightarrow \infty} n\left|c_{n}\right|^{1 / n}=e \sigma \quad(\text { see }[5]) .
$$

It follows that

$$
\varlimsup_{n \rightarrow \infty}\left|c_{n} \Gamma(n+r+1)\right|^{1 / n}=\sigma .
$$

Therefore,

$$
\frac{1}{\Gamma(r+1)} \sum_{n=0}^{\infty} \frac{c_{n} \Gamma(n+r+1)}{z^{n+r+1}}
$$

represents an analytic function in the region $\Omega \backslash(-\infty, 0)$, where $\Omega=\{z \in$ $\mathbb{C}:|z|>\sigma\}$.

Since $\Lambda_{z}^{r} V$ is analytic in the region $\operatorname{Re} z>0$ and $\Lambda_{z}^{r} V=\frac{1}{\Gamma(r+1)} \sum_{n=0}^{\infty}$ $\frac{c_{n} \Gamma(n+r+1)}{z^{n+r+1}}$ in the region $\{z \in \Omega: \operatorname{Re} z>0\}$, it follows that $\Lambda_{z}^{r} V$ can be analytically extended to the region $\mathbb{C} \backslash \mathcal{A}_{\sigma}$.

Corollary 3.6. Let $V \in \beta_{c}(\mathbb{R})$ such that supp $V=\{0\}$. Then, $\Lambda_{z}^{r} V$ can be analytically extended to the region $\mathbb{C} \backslash(-\infty, 0]$. Moreover,

$$
\Lambda_{z}^{r} V=\frac{1}{\Gamma(r+1)} \sum_{n=0}^{\infty} \frac{c_{n}}{z^{n+r+1}}, z \in \mathbb{C} \backslash(-\infty, 0],
$$

where $c_{n}=\frac{\widehat{V}^{(n)}(0)}{n !} \Gamma(n+r+1), n=0,1,2, \ldots$.

\section{Remarks 3.7.}

1. In the previous theorem and corollary, if $r \in \mathbb{N} \cup\{0\}$, then $\Lambda_{z}^{r} V$ can be analytically extended to the region $\mathbb{C} \backslash \tilde{B}_{\sigma}(0)$. 
2. For $V \in \beta_{c}(\mathbb{R})\left(W \in B_{r}(\mathbb{R})\right), \Lambda_{z}^{r} V\left(\Lambda_{z}^{r} W\right)$ will denote the Stieltjes transform of $V(W)$ as well as its extension. This should not cause any confusion.

The following example illustrates that the containment $J^{\prime}(r) \subset B_{r}(\mathbb{R})$ is proper.

Example 3.8. Let $W=\delta$ - $\lim _{n \rightarrow \infty} \sum_{k=0}^{n} \frac{D^{k} \delta}{(k !)^{2}}$ (for the convergence of the series, see [10]). Then, $W \in B_{r}(\mathbb{R}) \backslash J^{\prime}(r)$, and using the fact that $\operatorname{supp} W=$ $\{0\}$ and $\widehat{W}(t)=\sum_{n=0}^{\infty} \frac{t^{n}}{(n !)^{2}}$, we obtain

$$
\Lambda_{z}^{r} W=\sum_{n=0}^{\infty} \frac{(r+1)_{n}}{(n !)^{2} z^{n+r+1}}, z \in \mathbb{C} \backslash(-\infty, 0],
$$

where $(r+1)_{n}=(r+1)(r+2) \ldots(r+n)$. In particular, if $r=k \in \mathbb{N} \cup\{0\}$, then

$$
\Lambda_{z}^{k} W=\frac{(-1)^{k}}{k !} \frac{d^{k}}{d z^{k}}\left(\frac{1}{z} \exp \left(\frac{1}{z}\right)\right), z \in \mathbb{C} \backslash\{0\} .
$$

Let $W \in B_{r}(\mathbb{R})$. Then, $W=V+D^{k} f$ with $V \in \beta_{c}(\mathbb{R}), f \in L_{\text {loc }}^{1}(\mathbb{R})$, supp $V$ $\subseteq[0, \sigma]$, supp $f \subseteq[\sigma, \infty)$, and $f(t) t^{-r-k+\gamma}$ bounded as $t \rightarrow \infty$ (for some $\gamma>0)$. Hence,

$$
\Lambda_{z}^{r} W=\Lambda_{z}^{r} V+\Lambda_{z}^{r} D^{k} f \text { in the half-plane } \operatorname{Re} z>0 .
$$

Since, in the half-plane $\operatorname{Re} z>0$, the Stieltjes transform of $D^{k} f$ as an element of $B_{r}(\mathbb{R})$ agrees with the Stieltjes transform of $D^{k} f$ as an element of $J^{\prime}(r), \Lambda_{z}^{r} D^{k} f$ has an analytic extension to the region $\mathbb{C} \backslash(-\infty, 0]$.

So, by Theorem 3.5 and the above, we obtain the following theorem.

Theorem 3.9. Let $W \in B_{r}(\mathbb{R})$. Then there exists $\sigma \geq 0$ such that $\Lambda_{z}^{r} W$ can be analytically extended to the region $\mathbb{C} \backslash \mathcal{A}_{\sigma}$.

The product of $t$ and a Boehmian $W=\left[\frac{f_{n}}{\varphi_{n}}\right]$ is defined by

$$
t\left[\frac{f_{n}}{\varphi_{n}}\right]=\left[\frac{\left(t f_{n}\right) * \varphi_{n}-f_{n} *\left(t \varphi_{n}\right)}{\varphi_{n} * \varphi_{n}}\right] .
$$

For $n \in \mathbb{N}, t^{n} W$ is defined by induction. This definition is consistent with the product of $t^{n}$ and a function or distribution.

Properties 3.10. Let $W \in B_{r}(\mathbb{R})$. Then for $r>-1$ and $\operatorname{Re} z>0$,

1. $\Lambda_{z}^{r} \tau_{c} W=\Lambda_{z+c}^{r} W, c>0$.

2. $\Lambda_{z}^{r} D^{m} W=\frac{\Gamma(r+m+1)}{\Gamma(r+1)} \Lambda_{z}^{r+m} W, m=1,2, \ldots$.

3. $\frac{d^{m}}{d z^{m}} \Lambda_{z}^{r} W=\frac{(-1)^{m} \Gamma(r+m+1)}{\Gamma(r+1)} \Lambda_{z}^{r+m} W=(-1)^{m} \Lambda_{z}^{r} D^{m} W, m=1,2, \ldots$. 
4. $\Lambda_{z}^{r+n}\left(t^{n} W\right)=\sum_{k=0}^{n}(-1)^{k}\left(\begin{array}{c}n \\ k\end{array}\right) z^{k} \Lambda_{z}^{r+k} W, n=1,2, \ldots$.

The proofs of properties 1-3 follow directly from the properties of the Laplace transform.

Property 4: If $W \in B_{r}(\mathbb{R})$, it follows that $t W \in B_{r+1}(\mathbb{R})$, and using the properties of the Laplace transform, for $r>-1$ and $\operatorname{Re} z>0$,

$$
\Lambda_{z}^{r+1}(t W)=\Lambda_{z}^{r} W-z \Lambda_{z}^{r+1} W
$$

By using an inductive argument, for $n \in \mathbb{N}, t^{n} W \in B_{r+n}(\mathbb{R})$ and

$$
\Lambda_{z}^{r+n}\left(t^{n} W\right)=\sum_{k=0}^{n}(-1)^{k}\left(\begin{array}{l}
n \\
k
\end{array}\right) z^{k} \Lambda_{z}^{r+k} W, \text { Re } z>0 .
$$

\section{INVERSION}

We are now going to discuss inversion of the Stieltjes transform. But first, we prove an inversion formula for the Laplace transform.

Theorem 4.1. Let $W \in B_{r}(\mathbb{R})$ and $\gamma>0$. Then,

$$
W=\delta-\lim _{n \rightarrow \infty} \frac{1}{2 \pi i} \int_{\gamma-i n}^{\gamma+i n} e^{z t} \widehat{W}(z) d z
$$

Proof. $W=V+D^{k} f$, where $V=\left[\frac{h_{n}}{\psi_{n}}\right] \in \beta_{c}^{+}(\mathbb{R}), \operatorname{supp} h_{n} \subset[-a, a](n \in \mathbb{N})$ for some $a>0$, and $f$ has properties as previously stated.

So, $W=\left[\frac{f_{n}}{\varphi_{n}}\right]$, where $f_{n}=\left(h_{n}+D^{k} f * \psi_{n}\right) * \psi_{n}$ and $\varphi_{n}=\psi_{n} * \psi_{n}, n \in \mathbb{N}$. Moreover, for each $n \in \mathbb{N}$, supp $f_{n} \subset[-b, \infty)$ for some $b>0, f_{n} \in C^{\infty}(\mathbb{R})$, and $\left|f_{n}^{\prime \prime}(t)\right| \leq p_{n}(t)(t \in \mathbb{R})$, for some polynomial $p_{n}$.

Thus, for each $n \in \mathbb{N}$, there exists $M_{n}>0$ such that

$$
\left|\widehat{f}_{n}(z)\right| \leq \frac{M_{n}}{|z|^{2}}, \quad \text { for } z=\gamma+i y,
$$

where $y \in \mathbb{R}$ and $M_{n}$ is independent of $y$.

For $n=1,2, \ldots$, let

$$
g_{n}(t)=\frac{1}{2 \pi i} \int_{\gamma-i n}^{\gamma+i n} e^{z t} \widehat{W}(z) d z .
$$

Thus, $g_{n} \in C(\mathbb{R}) \subset \beta(\mathbb{R}), n=1,2, \ldots$. Now, using Fubini's theorem, for each $n$ and $k$ we obtain

$$
\left(g_{n} * \varphi_{k}\right)(t)=\frac{1}{2 \pi i} \int_{\gamma-i n}^{\gamma+i n} e^{z t} \widehat{W}(z) \widehat{\varphi}_{k}(z) d z=\frac{1}{2 \pi i} \int_{\gamma-i n}^{\gamma+i n} e^{z t} \widehat{f}_{k}(z) d z .
$$


Using (4.1) and the classical inversion formula for the Laplace transform [4], we obtain

$$
\frac{1}{2 \pi i} \int_{\gamma-i n}^{\gamma+i n} e^{z t} \widehat{f}_{k}(z) d z \rightarrow f_{k} \text { uniformly on compact sets as } n \rightarrow \infty .
$$

Thus, $\delta-\lim _{n \rightarrow \infty} \frac{1}{2 \pi i} \int_{\gamma-i n}^{\gamma+i n} e^{z t} \widehat{W}(z) d z=W$.

Now, we turn to the inversion for the Stieltjes transform.

Since inversion formulae for the classical Laplace transform are wellknown, it is possible to invert the Stieltjes transform. Roughly speaking, this can be accomplished by applying the classical inversion formula for the Laplace transform [4] to the Stieltjes transform of $W$, followed by the inversion formula for the Laplace transform of a Boehmian. More precisely, let $W \in B_{r}(\mathbb{R}), \gamma>0$, and $\tilde{U}(z)$ denote the analytic continuation of $U(t)$ to the half-plane $\operatorname{Re} z>0$, where $U(t)=\frac{1}{2 \pi i} \int_{\gamma-i \infty}^{\gamma+i \infty} e^{z t} \Lambda_{z}^{r} W d z$. Then, for any $\sigma>0$,

$$
W=\delta-\lim _{n \rightarrow \infty} \frac{\Gamma(r+1)}{2 \pi i} \int_{\sigma-i n}^{\sigma+i n} e^{z t} \frac{\tilde{U}(z)}{z^{r}} d z
$$

\section{Abelian theorems}

In this section, we establish theorems which describe the behavior of the Stieltjes transform of a Boehmian at the origin (infinity) in a wedge in the half-plane Re $z>0$ from the behavior of the Boehmian at the origin (infinity) along the real line.

Let $W \in \beta^{+}(\mathbb{R})$ and $\nu \neq-1,-2, \ldots$ Then, $W(t) \stackrel{e}{\sim} \xi t^{\nu}(\xi \in \mathbb{C})$ as $t \rightarrow 0^{+}(t \rightarrow \infty)$ provided there exist $a<0$ and $b>0 \quad(a>0$ and $b=\infty)$, $m \in \mathbb{N}, g \in L_{\text {loc }}^{1}(\mathbb{R})$ such that $W(t)=D^{m} g(t)$ on $(a, b)$ and $\frac{g(t)}{t^{m+\nu}} \rightarrow \frac{\xi}{(\nu+1)_{m}}$ as $t \rightarrow 0^{+}(t \rightarrow \infty)$.

Lemma 5.1. Let $V \in \beta_{c}^{+}(\mathbb{R})$ such that supp $V \subseteq[a, b]$, where $a>0$. Then, for $r>-1, \Lambda_{z}^{r} V$ is analytic in a neighborhood of the origin.

Proof. Let $U=\tau_{-a} V$. Then, $U \in \beta_{c}^{+}(\mathbb{R})$. So, by Remark $3.2(3)$, for each $\varepsilon>0, \widehat{U}(t)=O\left(e^{\varepsilon t}\right)$ as $t \rightarrow \infty$. Using this and the fact that $\widehat{V}(t)=e^{-a t} \widehat{U}(t)$, we see that for each $\varepsilon>0, \widehat{V}(t)=O\left(e^{-(a-\varepsilon) t}\right)$ as $t \rightarrow \infty$. Therefore, for $r>-1, t^{r} \widehat{V}(t)$ is locally integrable and for each $\varepsilon>0, t^{r} \widehat{V}(t)=O\left(e^{-(a-\varepsilon) t}\right)$ as $t \rightarrow \infty$. Thus,

$\Lambda_{z}^{r} V=\frac{1}{\Gamma(r+1)} \int_{0}^{\infty} e^{-z t} t^{r} \widehat{V}(t) d t$ is analytic in the half-plane $\operatorname{Re} z>-a$. 
Theorem 5.2. (Initial Value Theorem). Let $W \in B_{r}(\mathbb{R})$ and $\nu>-1$. If $W(t) \stackrel{e}{\sim} \xi t^{\nu}$ as $t \rightarrow 0^{+}$, then for $r>\nu$

$$
\lim _{\substack{z \rightarrow 0 \\|\arg z| \leq \psi<\pi / 2}} \frac{z^{r-\nu} \Gamma(r+1) \Lambda_{z}^{r} W}{\Gamma(r-\nu) \Gamma(\nu+1)}=\xi .
$$

Proof. There exist $m, k \in \mathbb{N}$ such that

$$
W=D^{m} g+V+D^{k} f
$$

where $V \in \beta_{c}^{+}(\mathbb{R})$ and $f, g \in L_{l o c}^{1}(\mathbb{R})$ with $\operatorname{supp} g \subseteq[a, b], \operatorname{supp} V \subseteq$ $[b, c], \operatorname{supp} f \subseteq[c, \infty)(a<0<b \leq c), \frac{f(t)}{t^{r+k-\alpha}}$ is bounded on $[c, \infty)$ (some $\alpha>0)$, and $\frac{g(t)}{t^{m+\nu}} \rightarrow \frac{\xi}{(\nu+1)_{m}}$ as $t \rightarrow 0^{+}$.

Thus,

$$
z^{r-\nu} \Lambda_{z}^{r} W=z^{r-\nu} \Lambda_{z}^{r} D^{m} g+z^{r-\nu} \Lambda_{z}^{r} V+z^{r-\nu} \Lambda_{z}^{r} D^{k} f
$$

By using the Initial Value Theorem for functions (see [2], Theorem 1), it follows that

$$
\lim _{\substack{z \rightarrow 0 \\|\arg z| \leq \psi<\pi / 2}} \frac{z^{r-\nu} \Gamma(r+m+1) \Lambda_{z}^{r+m} g}{\Gamma(r-\nu) \Gamma(m+\nu+1)}=\frac{\xi}{(\nu+1)_{m}}
$$

Now, by (5.2) and

$$
\frac{z^{r-\nu} \Gamma(r+1) \Lambda_{z}^{r} D^{m} g}{\Gamma(r-\nu) \Gamma(\nu+1)}=\frac{z^{r-\nu} \Gamma(r+m+1) \Lambda_{z}^{r+m} g}{\Gamma(r-\nu) \Gamma(\nu+1)},
$$

we obtain

$$
\lim _{\substack{z \rightarrow 0 \\|\arg z| \leq \psi<\pi / 2}} \frac{z^{r-\nu} \Gamma(r+1) \Lambda_{z}^{r} D^{m} g}{\Gamma(r-\nu) \Gamma(\nu+1)}=\xi
$$

Now,

$$
\lim _{\substack{z \rightarrow 0 \\|\arg z| \leq \psi<\pi / 2}} \frac{z^{r-\nu} \Gamma(r+1) \Lambda_{z}^{r} D^{k} f}{\Gamma(r-\nu) \Gamma(\nu+1)}=0 \quad \text { (see [3]) }
$$

By the previous lemma, we obtain

$$
\lim _{\substack{z \rightarrow 0 \\|\arg z| \leq \psi<\pi / 2}} \frac{z^{r-\nu} \Gamma(r+1) \Lambda_{z}^{r} V}{\Gamma(r-\nu) \Gamma(\nu+1)}=0
$$

The desired result follows by combining (5.1), (5.3), (5.4), and (5.5).

Theorem 5.3. (Final Value Theorem). Let $W \in \beta^{+}(\mathbb{R})$ and $\nu>-1$. If $W(t) \stackrel{e}{\sim} \xi t^{\nu}$ as $t \rightarrow \infty$, then for $r>\nu$,

$$
\lim _{\substack{z \rightarrow \infty \\|\arg z| \leq \psi<\pi / 2}} \frac{z^{r-\nu} \Gamma(r+1) \Lambda_{z}^{r} W}{\Gamma(r-\nu) \Gamma(\nu+1)}=\xi .
$$


Proof. There exist $m \in \mathbb{N}, a \in \mathbb{R}$, and $g \in L_{\text {loc }}^{1}(\mathbb{R})$ such that $W(t)=D^{m} g(t)$ on $(a, \infty)$ and $\lim _{t \rightarrow \infty} \frac{g(t)}{t^{m+\nu}}=\frac{\xi}{(\nu+1)_{m}}$. Thus,

$$
W=V+D^{m} g \text {, }
$$

where $V \in \beta_{c}^{+}(\mathbb{R})$. Now,

$$
\lim _{\substack{z \rightarrow \infty \\|\arg z| \leq \psi<\pi / 2}} \frac{z^{r-\nu} \Gamma(r+1) \Lambda_{z}^{r} D^{m} g}{\Gamma(r-\nu) \Gamma(\nu+1)}=\xi \quad \text { (see [3]). }
$$

Since $V \in \beta_{c}^{+}(\mathbb{R})$, there exist $\sigma>0$ and $c_{n} \in \mathbb{C}(n \in \mathbb{N})$ such that for $r>\nu>-1$ and $\operatorname{Re} z>\sigma$,

$$
z^{r-\nu} \Lambda_{z}^{r} V=\frac{1}{\Gamma(r+1) z^{\nu+1}} \sum_{n=0}^{\infty} \frac{c_{n} \Gamma(n+r+1)}{z^{n}}
$$

(see proof of Theorem 3.5). Therefore,

$$
\lim _{\substack{z \rightarrow \infty \\|\arg z| \leq \psi<\pi / 2}} \frac{z^{r-\nu} \Gamma(r+1) \Lambda_{z}^{r} V}{\Gamma(r-\nu) \Gamma(\nu+1)}=0 .
$$

Thus, by (5.6), (5.7), and (5.8) the proof is complete.

\section{Open PROBLEMS}

1. Since the Stieltjes transform for a distribution is an analytic function in the region $C \backslash(-\infty, 0]$ [9], is it possible to improve Theorem 3.9?

Conjecture: Given $W \in B_{r}(\mathbb{R})$. The Stieltjes transform of $W$ can be analytically extended to $\mathbb{C} \backslash(-\infty, 0]$.

2. Is it possible to give a characterization for the range of the operator $\Lambda_{(\cdot)}^{r}$ ? That is, is a Paley-Wiener type theorem possible?

\section{REFERENCES}

[1] Yu. A. Brychkov and A.P. Prudnikov, Integral Transforms of Generalized Functions, Gordon and Breach Science Publishers, Amsterdam, 1989.

[2] R.D. Carmichael, Abelian Theorems for the Stieltjes Transform of Functions, Bull. Cal. Math. Soc., 68 (1976), 49-52.

[3] R. D. Carmichael and E. O. Milton, Abelian theorems for the distributional Stieltjes transform, J. Math. Anal. Appl., 72 (1979), 195-205.

[4] G. Doetsch, Theorie der Laplace Transformation (Band I), Verlag Birkhauser, Basel,1950.

[5] A. S. B. Holland, Introduction to the Theory of Entire Functions, Pure and Applied Mathematics; a series of monographs and textbooks, v. 56, New York, Academic press, 1973. 
[6] J. L. Lavoine and O. P. Misra, Sur la transformation de Stieltjes des distributions et son inversion au Moyen de la transformation de Laplace, C.R. Acad. Sc. Paris, 290 (1980), 139-142.

[7] V. Marić, M. Skendžić, and A. Takači, On Stieltjes transform of distributions behaving as regularly varying functions, Acta Sci. Math. (Szoged), 50 (1986), 405-410.

[8] P. Mikusiński, A. Morse, and D. Nemzer, The two-sided Laplace transform for Boehmians, Integral Transforms Spec. Func., 2 (1994), 219-230.

[9] O. P. Misra and J. L. Lavoine, Transform Analysis of Generalized Functions, Elsevier Science Publishers, Amsterdam, 1986.

[10] D. Nemzer, A note on the convergence of a series in the space of Boehmians, Bull. Pure Appl. Math., 2 (2008), 63-69.

[11] D. Nikolić-Despotović and S. Pilipović, Abelian Theorem for the Distributional Stieltjes Transformation, Generalized Functions, Convergence Structures and their Applications, Plenum Press, New York and London, 1988, 269-277.

[12] S. Pilipović, B. Stanković, and A. Takači, Asymptotic Behaviour and Stieltjes Transformation of Distributions, Taubner, Leipzig, 1990.

[13] R. Roopkumar, Stieltjes transform for Boehmians, Integral Transforms Spec. Func., 18 (2007), 819-827.

[14] R. Roopkumar and E. R. Negrin, A unified extension of Stieltjes and Poisson transforms, Integral Transforms Spec. Func., 22 (2011), 195-206.

[15] T. S. Stieltjes, Recherches sur les fractions continues, Annales de la Faculte des Sciences de Toulouse, 8 (1894), 1-123.

[16] A. H. Zemanian, Distribution Theory and Transform Analysis, Dover Publications, New York, 1987.

(Received: November 16, 2013)

Department of Mathematics California State University, Stanislaus One University Circle Turlock, CA 95382

U.S.A.

nemzer@comcast.net 